\title{
Efectividad del uso de ketamina perioperatoria en pacientes sometidos a cirugía de raquis en tratamiento crónico con opioides
}

\author{
Postoperative ketamine efficacy in patients receiving chronic \\ opioids undergoing spinal surgery
}

\author{
U. Rodríguez Rivas, E. Vilà Barriuso, L. Trillo Urrutia, M. Soldevilla García y J. García Álvarez
}

Servicio de Anestesiología y Reanimación. Parc de Salut Mar. Barcelona, España

\section{RESUMEN}

Introducción: El número de pacientes en tratamiento con opioides de forma crónica sometidos a una cirugía espinal ha aumentado en los últimos años. Los registros de dolor mediante la escala visual analógica (EVA) y el consumo de opioides durante el postoperatorio son más elevados en esta clase de pacientes.

Material y métodos: Siguiendo las recomendaciones de la American Pain Society (APS), en 2018 se diseñó un nuevo protocolo analgésico intra y postoperatorio basado en el uso de ketamina. En el intraoperatorio, se administró un bolus de $0,5 \mathrm{mg} / \mathrm{kg}$ de ketamina, seguido por una perfusión a dosis de $0,2 \mathrm{mg} / \mathrm{kg} / \mathrm{h}$ hasta el cierre de la herida. Durante las 48 horas postoperatorias, se mantuvo una bomba de analgesia controlada por el paciente (PCA) de morfina-ketamina junto con un régimen de analgesia multimodal con paracetamol y dexketoprofeno. Se realizó un análisis de la efectividad del nuevo protocolo (grupo ketamina) comparándolo con el protocolo seguido el año anterior (grupo control), basado en el uso de tramadol $100 \mathrm{mg} / 6 \mathrm{~h}$ o PCA de morfina. Se analizaron los registros de dolor mediante la escala verbal numérica (EVN) durante las primeras 48 horas postoperatorias, la necesidad de administración de bolus puntuales de morfina y la necesidad de iniciar una perfusión continua de morfina (grupo control) o morfina ketamina (grupo ketamina).

Resultados: Los pacientes del grupo ketamina presentaron EVN inferiores a los del grupo control durante las dos primeras horas postoperatorias ( $p=0,001)$ y menores necesidades de rescates de morfina en el

\section{ABSTRACT}

Introduction: The number of patients receiving chronic opioids undergoing spinal surgery has increased lately. Elevate records in visual analog scale (VAS) and opioid consumption are higher in this group of patients.

Material and methods: Following the recommendations of the American Pain Society (APS), a new intra and postoperative analgesic protocol based on the use of ketamine was designed in 2018. Intraoperatively, a bolus of $0.5 \mathrm{mg} / \mathrm{kg}$ of ketamine was administered, followed by a dose infusion of $0.2 \mathrm{mg} / \mathrm{kg} / \mathrm{h}$ until the surgical wound was closed. During the first 48 postoperative hours, a patient-controlled analgesia pump (PCA) of morphine-ketamine was maintained along with a multimodal analgesia regimen with paracetamol and dexketoprofen. An effectiveness analysis comparing the new protocol (ketamine group) with the previous one (control group), based on the use of tramadol $100 \mathrm{mg} / \mathrm{6h}$ or PCA morphine, was done. During the first 48 postoperative hours, NVS records, need of rescue analgesia, morphine bolus or continuous morphine infusion (control group) or ketamine morphine infusion (ketamine group) were analyzed.

Results: The patients in the ketamine group had lower NVS records than those in the control group during the first two postoperative hours $[p=0.001)$ and lower morphine rescues needs on the second postoperative day ( $p=0.003$ ). The need for continuous morphine-ketamine perfusion was significantly lower than the need for continuous morphine perfusion on the control group $(p=0.011)$.
Rodríguez Rivas U, Vilà Barriuso E, Trillo Urrutia L, Soldevilla García M, García Álvarez J. Efectividad del uso de ketamina perioperatoria en pacientes sometidos a cirugía de raquis en tratamiento crónico con opioides. Rev Soc Esp Dolor. 2021;28[2]:92-99
Recibido: 16-06-2020

Aceptado: 22-03-2021

Correspondencia: Uxía Rodríguez Rivas 60533@parcdesalutmar.cat 
segundo día postoperatorio $(p=0,003)$. La necesidad de perfusión continua de morfina-ketamina fue significativamente inferior a la necesidad de inicio de perfusión continua de morfina en el grupo control ( $p=0,011)$.

Conclusión: El protocolo basado en el uso de ketamina consiguió mejorar el control del dolor postoperatorio y reducir de forma significativa el consumo de opioides en las primeras 48 horas tras la intervención.

Palabras clave: Dolor, postoperatorio, ketamina, cirugía, espinal.
Conclusion: The protocol based on the use of ketamine, managed to improve the control of postoperative pain and significantly reduce the consumption of opioids in the first 48 hours after the intervention.

Key words: Pain, postoperative, ketamine, surgery, spine.

\section{INTRODUCCIÓN}

Los pacientes sometidos a cirugía de raquis experimentan dolor moderado-intenso durante el postoperatorio (1). Los registros de intensidad del dolor mediante EVA suelen oscilar entre 5 y 8 , dependiendo del procedimiento realizado $(1,2)$. En nuestro centro, un $20 \%$ de los pacientes intervenidos están recibiendo tratamiento con opioides de forma crónica antes de la cirugía. Esta incidencia es similar a la descrita en la literatura $[3,4]$.

En las primeras $48 \mathrm{~h}$ postoperatorias, los pacientes en tratamiento crónico con opioides presentan requerimientos de morfina y registros de EVA más elevados (5). Se recomienda el uso de fármacos adyuvantes no opioides [6] para optimizar su tratamiento analgésico. La ketamina a dosis bajas es útil en este contexto $[6,7]$.

En el año 2018, siguiendo las guías de la APS [6], en nuestro centro se decidió cambiar el protocolo analgésico postoperatorio para pacientes en tratamiento crónico con opioides programados para cirugía de columna. En el periodo intraoperatorio se protocolizó el uso de una perfusión continua (PC) de ketamina a dosis bajas. En las primeras 48 horas postoperatorias, se optó por el uso de un PCA con morfina-ketamina en sustitución del protocolo empleado hasta ese momento; en el que se pautaba tramadol ( $100 \mathrm{mg} / 6 \mathrm{~h}$ ) o una PCA con morfina intravenosa. En ambos protocolos, además, se contempla una analgesia multimodal con paracetamol y dexketoprofeno pautados.

Los objetivos principales de este análisis fueron comparar la intensidad del dolor evaluada mediante EVN y el consumo de opioides de rescate en las primeras 48 horas postoperatorias entre dos cohortes de pacientes, una formada por los pacientes que recibieron tramadol o PCA de morfina (grupo control) y otra formada por pacientes que recibieron ketamina intraoperatoria con una pauta de PCA con morfina-ketamina (grupo ketamina) en las primeras 48 horas postoperatorias.

\section{MATERIAL Y MÉTODOS}

Análisis de eficacia retrospectivo del nuevo protocolo analgésico implementado en el servicio de Anestesiología del Hospital del Mar de Barcelona, comparado con el protocolo previo. Ningún dato confidencial de la historia clínica de los pacientes se publica.

\section{Criterios de inclusión y exclusión}

Se incluyeron en el análisis a todos los pacientes en tratamiento con opioides fuertes o dosis de tramadol oral iguales o superiores a $200 \mathrm{mg} / 24 \mathrm{~h}$, durante 6 semanas o más, antes de someterse a cualquier tipo de cirugía de espalda durante los años 2017 y 2018.

Se excluyeron a los pacientes que no completaron de forma correcta el protocolo analgésico.

- Protocolo analgésico aplicado al grupo control [año 2017): paracetamol $1 \mathrm{~g} / 6 \mathrm{~h}$ i.v., dexketoprofeno $50 \mathrm{mg} / 12$ h i.v., tramadol $100 \mathrm{mg} / 6$ h i.v. o PCA de morfina $1 \mathrm{mg} / \mathrm{ml}$ (bolus de $1 \mathrm{ml}$, tiempo de cierre 10 min. Máximo 6 bolus/h) según el criterio del anestesiólogo responsable.

Analgesia de rescate en la unidad de críticos postquirúrgicos (UCPQ): bolus de $2 \mathrm{mg}$ de morfina i.v. hasta obtener una EVN < 3 como primera opción, o inicio de perfusión continua de morfina a $1 \mathrm{ml} / \mathrm{h}$ como segunda opción.

Analgesia de rescate en la unidad de hospitalización: bolus de morfina subcutánea [s.c.]/4 h a dosis de 0,01 a 0,05 mg/kg hasta alcanzar una EVN < 3 .

- Protocolo analgésico aplicado al grupo ketamina [año 2018): bolus de ketamina a dosis de $0,5 \mathrm{mg} / \mathrm{kg}$ en la inducción anestésica, seguido de perfusión continua a dosis de 0,2 mg/ $\mathrm{kg} / \mathrm{h}$ hasta el cierre de la herida quirúrgica. En el periodo postoperatorio, se administraron: paracetamol $1 \mathrm{~g} / 6 \mathrm{~h}$ i.v., dexketoprofeno $50 \mathrm{mg} / 8 \mathrm{~h}$ i.v., y PCA morfina-ketamina durante 48 h lsiendo la preparación de la misma con una ratio $1 \mathrm{mg} / 1 \mathrm{mg}$, bolus de $1 \mathrm{ml}$, tiempo de cierre 10 min., máximo 6 bolus/h).

Analgesia de rescate en la UCPQ: bolus de $2 \mathrm{mg}$ de morfina i.v. hasta alcanzar una EVN < 3 como primera opción, o inicio de perfusión continua de morfina-ketamina a $1 \mathrm{ml} / \mathrm{h}$ como segunda opción.

Analgesia de rescate en la unidad de hospitalización: bolus de morfina s.c. /4 h a dosis de 0,01 a $0,05 \mathrm{mg} / \mathrm{kg}$ hasta alcanzar una $\mathrm{EVN}<3$. 


\section{Variables evaluadas}

Todos los datos se recogieron de los registros médicos y de enfermería de la historia clínica informatizada de los pacientes. Se analizaron los siguientes datos demográficos: sexo, edad y diagnóstico prequirúrgico. Sobre el tratamiento analgésico preoperatorio se recogieron: el tipo de opioide y la dosis administrada, el uso de medicación concomitante y la realización de técnicas analgésicas invasivas. Sobre la intervención quirúrgica se recogieron los siguientes datos: técnica quirúrgica realizada (laminectomía, artrodesis, reartrodesis, extracción de material, etc.), tiempo quirúrgico, nivel y espacios intervenidos y opioide intraoperatorio utilizado. Durante el periodo postquirúrgico se recogieron los registros de EVN a las $2 \mathrm{~h}$ tras el fin de la cirugía, y durante $48 \mathrm{~h}$ del postoperatorio (evaluados cada $8 \mathrm{~h}$ ), el tipo de opioide pautado, su dosis y la administración de analgesia de rescate. También se documentaron la aparición de síntomas psicomiméticos, alteración del nivel de conciencia, depresión respiratoria y náuseas o vómitos.

\section{Análisis estadístico}

Para el análisis estadístico se utilizó el paquete estadístico SPSS versión 17 para Windows. En la descripción de variables continuas se utilizó la media y la desviación estándar. Para la descripción de variables categóricas se utilizó el número y porcentaje de pacientes por categoría de respuesta. Se utilizaron técnicas estadísticas para asegurar el cumplimiento de los supuestos estadísticos, previas a la realización de las pruebas comparativas. En caso de que no se cumplan los supuestos establecidos, se utilizaron las pruebas no paramétricas correspondientes. Los análisis comparativos se realizaron únicamente para variables de tipo categórico. Se aplicó la prueba Chi cuadrado en las comparaciones con variable dependiente nominal y la $\mathrm{U}$ de Mann-Whitney en las comparaciones con variable dependiente ordinal. En todas las pruebas estadísticas realizadas se utilizó un nivel de significación estadística de 0,05.

\section{RESULTADOS}

En el grupo control se incluyeron 43 pacientes. En el grupo ketamina, se incluyeron 37 de los 47 pacientes en tratamiento crónico con opioides intervenidos en 2018. Se excluyeron 10: en 3 no se aplicó el protocolo morfina-ketamina (M-K) y en 7 no se aplicó en su totalidad (Figura 1).

Las características demográficas son similares en ambos grupos (Tabla I). Los diagnósticos prequirúrgicos más frecuentes fueron estenosis de canal y hernia discal. La técnica quirúrgica más frecuente fue la artrodesis. El $50 \%$ de los pacientes se sometían por primera vez a una cirugía espinal.

En los dos grupos analizados (tramadol, tapentadol y fentanilo transdérmico) fueron los opioides más pautados preoperatoriamente. El porcentaje de pacientes que recibían tratamiento concomitante con dos fármacos

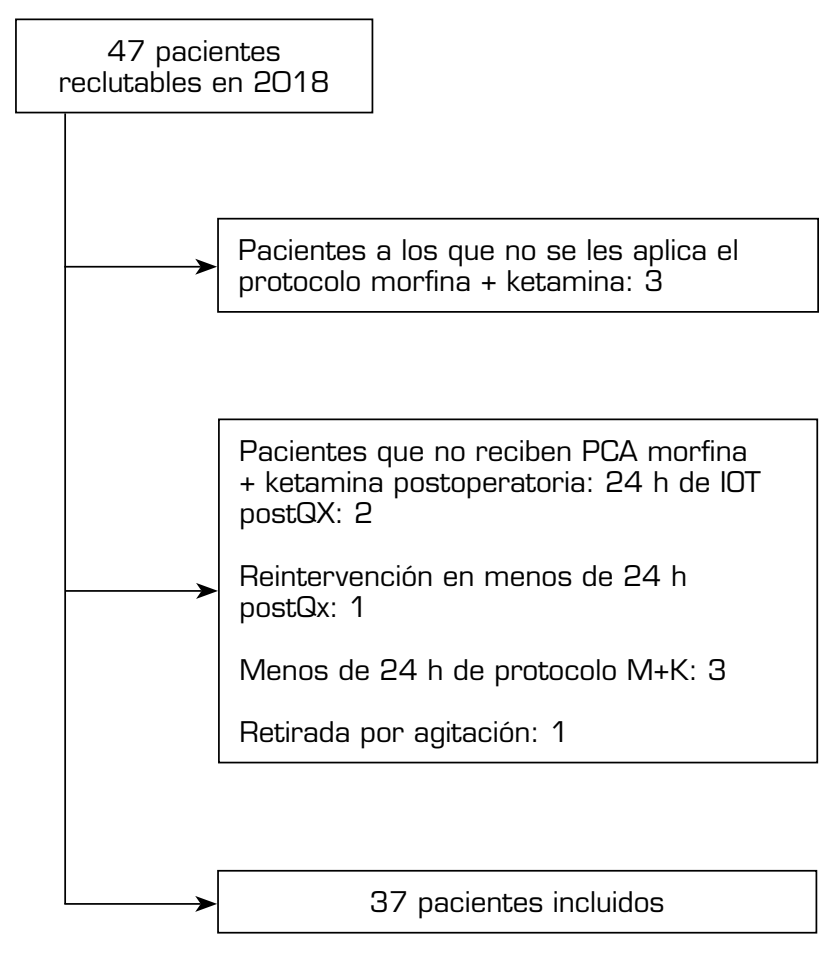

Fig. 1. Flow-chart. Pacientes reclutables e incluidos.

opioides fue similar en ambos grupos: control 16,27 \%, ketamina 13,51\% (Tabla II).

La dosis media de morfina preoperatoria se expresa en miliequivalentes ( $\mathrm{mEq}$ ) orales de morfina y fue calculada según las fuentes del Anexo I. En el grupo control, los pacientes recibían una dosis media de 75,93 $\mathrm{mEq}$, con una desviación estándar (DS) de 59,97mEq. En el grupo ketamina, la media fue de $50 \mathrm{mEq}$ (DS 71,60mEq).

Preoperatoriamente, todos los pacientes analizados realizaban analgesia multimodal combinando, al menos, un opioide con otro fármaco analgésico (Tabla III). En ambos grupos, más de un tercio recibían medicación para el control del dolor neuropático.

La diferencia en el uso de técnicas analgésicas invasivas (control $8,5 \%$ vs. ketamina $100 \%$ ) no es analizable. En la mayoría de los pacientes del grupo control (67\%), no se disponen de datos al respecto debido a la ausencia de registros en la historia clínica electrónica.

Durante el intraoperatorio, el fentanilo fue el opioide más utilizado (grupo ketamina 82,6\%, grupo control $93 \%$ ). Las dosis empleadas oscilaron entre los 400 y 1100 mcg en ambos grupos. La dosificación varía en función de la intervención realizada (por ejemplo: discectomía vs. artrodesis de múltiples niveles con osteotomías) y de los requerimientos de opioide intraoperatorios necesarios según el criterio de cada anestesiólogo responsable de la cirugía.

La Figura 2 muestra los registros de EVN durante las dos primeras horas del postoperatorio, de la hora 2 a la 24, y durante el segundo día del postoperatorio. Excepto en las primeras 2 horas postquirúrgicas, durante las cuales se reducen los registros EVN del grupo ketamina $(p=0,001)$, no existen diferencias entre los dos grupos. La Figura 3 muestra la necesidad 
TABLA I

DATOS DEMOGRÁFICOS

\begin{tabular}{|c|c|c|}
\hline & Control & Ketamina \\
\hline Número pacientes incluidos & 43 & 37 \\
\hline Sexo $(H / M)$ & $18 / 25$ & $16 / 21$ \\
\hline Edad media (DS) & $64(12,70)$ & $64(12,60)$ \\
\hline \multicolumn{3}{|l|}{ Diagnósticos preoperatorios* } \\
\hline Infección herida quirúrgica & $0,00 \%$ & $3(8.10 \%)$ \\
\hline Infección material protésico & $0,00 \%$ & $0,00 \%$ \\
\hline Pérdida de eje axial & $3(6,98 \%)$ & $5(13,51 \%)$ \\
\hline Espondilodiscitis & $0,00 \%$ & 9 (24,32\%) \\
\hline Hernia discal & $11[25,58 \%]$ & $16[43,24 \%]$ \\
\hline Compresión medular & $0,00 \%$ & 3 [8,10\%] \\
\hline Compresión radicular & $0,00 \%$ & $6(16.21 \%)$ \\
\hline Estenosis de canal & $13(30,23 \%]$ & 19 [51.35\%] \\
\hline Espondilolistesis & $6(13,95 \%)$ & $5(16,21 \%)$ \\
\hline Sindrome postlaminectomía & 4 (9,3\%) & $6(16,21 \%) \%$ \\
\hline Aflojamiento material protésico & $3(6,98 \%)$ & $0,00 \%$ \\
\hline Pseudoartrosis & $3(6,98 \%)$ & $3(8,10 \%)$ \\
\hline Fractura vertebral traumática & $2(4,65 \%)$ & $1(2,70 \%)$ \\
\hline Fractura vertebral osteoporótica & $2[4,65 \%)$ & $0,00 \%$ \\
\hline \multicolumn{3}{|l|}{ Técnicas intraoperatorias * * } \\
\hline Artrodesis & $26(60,47 \%)$ & 22 [59.45\%] \\
\hline Artrodesis percutánea & 1 (2,326 \%) & $0,00 \%$ \\
\hline Reartrodesis & 11 [25,58\%] & $4(10,81 \%)$ \\
\hline Desbridamiento & $0,00 \%$ & 3 [8,10\%] \\
\hline Extracción de material & $6(13,95 \%)$ & $3[8,10 \%]$ \\
\hline Laminectomía & 12 [27,91\%] & $13[35,14 \%]$ \\
\hline Osteotomías de corrección & $1[2,33 \%]$ & $4(10,81 \%)$ \\
\hline Colocación caja intersomática & $0,00 \%$ & $5(10,81 \%)$ \\
\hline \multicolumn{3}{|l|}{ Intervenciones previas } \\
\hline 0 & 24 [56.81\%] & $19[51,35 \%]$ \\
\hline 1 & $12[27,96 \%]$ & $11[29,73 \%]$ \\
\hline Más de 1 & $7(16,27 \%)$ & $7(18,92 \%)$ \\
\hline
\end{tabular}

*Los diagnósticos no son excluyentes. ** Las técnicas quirúrgicas no son excluyentes.

de administración de bolus de morfina de rescate en los mismos periodos de tiempo. Existen diferencias significativas estadísticamente durante el segundo día postquirúrgico ( $p=0,003$ ) a favor del grupo ketamina.

De la historia clínica electrónica de los pacientes, solo se pudieron obtener datos de la dosis de los bolus de morfina administrados durante las primeras $24 \mathrm{~h}$ postquirúrgicas. En el grupo control, durante las 2 primeras h posquirúrgicas se administró una media de 2,47 mg de morfina i.v. (2-10 mg). Entre las 2 y las 24 primeras horas, se administró una media de 0,49 mg (0-10 mg). En el grupo ketamina, durante las 2 prime- ras horas se administró una media de 1,69 mg de morfina i.v. (0-8 mg). Entre las 2 y las 24 h, se administró una media de 0,62 mg [0-8 mg].

En cuanto a la necesidad de la instauración de una perfusión continua, bien de morfina en el grupo control o morfina-ketamina en el grupo ketamina, los pacientes del grupo ketamina presentaron necesidades significativamente menores (Figura 4).

Respecto a las complicaciones por el tratamiento analgésico, ninguno de los pacientes del grupo control presentó síntomas psicomiméticos, solo 1 presentó náuseas o vómitos postoperatorios (NVPO), 6 presen- 
TABLA II

TRATAMIENTO OPIOIDEO PREQUIRÚRGICO

\begin{tabular}{|c|c|c|}
\hline & Control & Ketamina \\
\hline Fentanilo transdérmico & $7(16,28 \%)$ & $13[35,13 \%]$ \\
\hline Hidromorfona & $3(6,98 \%)$ & $1(2,70 \%)$ \\
\hline Metadona & $2[4,65 \%)$ & $0,00 \%$ \\
\hline Morfina oral & $0,00 \%$ & $0,00 \%$ \\
\hline Morfina transmucosa oral & $0,00 \%$ & $0,00 \%$ \\
\hline Morfina transmucosa nasal & $0,00 \%$ & $0,00 \%$ \\
\hline Oxicodona & $5(11,63 \%)$ & $0,00 \%$ \\
\hline Oxicodona/Naloxona & $0,00 \%$ & $1(2,70 \%)$ \\
\hline Oxicodona Retard & $0,00 \%$ & $1[2,70 \%]$ \\
\hline Tapentadol & $14(32,56 \%]$ & $12[32,43 \%]$ \\
\hline Tramadol $\geq 200 \mathrm{mg} / 24 \mathrm{~h}$ & $12(27,91 \%)$ & 9 (24,32 \%) \\
\hline \multicolumn{3}{|c|}{ Uso de un segundo opioide } \\
\hline Número de pacientes (\%) & $7(16,27 \%)$ & $5(13,51 \%)$ \\
\hline Fentanilo transdérmico & $1(14,28 \%)$ & $3(60,00 \%)$ \\
\hline Hidromorfona & $0,00 \%$ & $0,00 \%$ \\
\hline Metadona & $0,00 \%$ & $1(20,00 \%)$ \\
\hline Morfina oral & $2[28,57 \%]$ & $0,00 \%$ \\
\hline Morfina transmucosa oral & $0,00 \%$ & $0,00 \%$ \\
\hline Morfina transmucosa nasal & $0,00 \%$ & $0,00 \%$ \\
\hline Oxicodona & $0,00 \%$ & $0,00 \%$ \\
\hline Oxicodona/Naloxona & $0,00 \%$ & $0,00 \%$ \\
\hline Oxicodona Retard & $0,00 \%$ & $0,00 \%$ \\
\hline Tapentadol & $2[28,57 \%]$ & $1(20,00 \%)$ \\
\hline Tramadol & $2(28,57 \%)$ & $1[20,00 \%]$ \\
\hline
\end{tabular}

taron disminución del nivel de conciencia (somnolencia) y 1 presentó bradipnea. En el grupo ketamina, 1 paciente presentó disforia y 3 pacientes presentaron NVPO. Ninguno de los pacientes del grupo ketamina presentó alteraciones respiratorias o del nivel de conciencia.

\section{DISCUSIÓN}

Con la implantación del protocolo analgésico basado en el uso de ketamina a dosis bajas en 2018 , hemos conseguido reducir la necesidad de opioides de rescate en nuestro centro y hemos mejorado el control del dolor en las dos primeras horas postoperatorias. No se ha registrado una mayor incidencia de eventos adversos.

El número de pacientes con dolor de espalda en tratamiento con opioides ha aumentado en las últimas décadas [8-10]. La analgesia multimodal es la forma de abordaje más correcta, tanto en el periodo pre como postoperatorio $[6,11]$. A dosis subanestésicas, la ketamina ha demostrado disminuir los requerimientos de opioides postoperatorios, especialmente en este tipo de pacientes $[6,7,12,13)$.
Los resultados observados en nuestro análisis son comparables con los resultados publicados por Loftus y cols. (14) y Nielsen y cols. (15). Ambos ensayos clínicos demostraron la reducción de consumo de opioides postoperatorios y mejoría del control del dolor en pacientes en tratamiento crónico con opioides.

Al igual que Nielsen y cols., decidimos incluir a los pacientes tratados con tramadol en el análisis. Aunque la dosis mínima opioides con la cual un paciente puede presentar tolerancia o hiperalgesia secundaria es incierta, se ha observado que dosis inferiores a $40 \mathrm{mg}$ de morfina oral pueden provocarlos [16].

En el estudio de Nielsen y cols. se realizó un subanálisis para dilucidar si los pacientes con dosis más altas de morfina en el periodo preoperatorio presentaban una mayor reducción del consumo de mórficos en el postoperatorio. Destaca que con dosis superiores a 36 mEq morfina oral, la reducción es mayor. En nuestro caso, no se realizó dicho análisis por el escaso número de pacientes incluidos.

El uso de opioides a altas dosis durante el intraoperatorio provoca hiperalgesia y tolerancia durante las dos primeras horas postquirúrgicas [17-19]. Sin embargo, 
TABLA III

OTROS FÁRMACOS ANALGÉSICOS Y TÉCNICAS ANALGÉSICAS INVASIVAS PREOPERATORIAS

\begin{tabular}{|c|c|c|}
\hline & Control & Ketamina \\
\hline Paracetamol & $17(39,53 \%)$ & $29[78,38 \%]$ \\
\hline AINE & $17(39,53 \%)$ & $16[43,24 \%]$ \\
\hline Gabapentina & $8[18,60 \%]$ & $10[27,03 \%]$ \\
\hline Pregabalina & $15[34,88 \%]$ & $10[27,03 \%]$ \\
\hline Antidepresivos (amitriptilina o duloxetina) & $4[9,30 \%]$ & $12[34,43 \%]$ \\
\hline Capsaicina & $0,00 \%$ & $0,00 \%$ \\
\hline Clonazepam/Rivotril & $3(6,98 \%)$ & $2[5,40 \%)$ \\
\hline Combinación tramadol-paracetamol & $0,00 \%$ & $1(2,70 \%)$ \\
\hline Lidocaína transdérmica & $1(2,33 \%)$ & $2[5,40 \%)$ \\
\hline \multicolumn{3}{|c|}{ Técnicas analgésicas invasivas } \\
\hline N. ${ }^{\circ}$ de pacientes a los que se realizaron & $4(9,30 \%)$ & 22 [59.48 \%] \\
\hline Foraminal & $1[25,00 \%)$ & $4(18,18 \%)$ \\
\hline Interlaminar & $0,00 \%$ & $13[59,09 \%]$ \\
\hline Rizolisis & $3(75,00 \%)$ & $6(27,02 \%)$ \\
\hline TENS & $0,00 \%$ & $1[4,54 \%)$ \\
\hline $\mathrm{N} .^{\circ}$ de pacientes a los que no se realizaron & 29 & 15 \\
\hline Sin datos al respecto & 10 & 0 \\
\hline
\end{tabular}

$\mathrm{p} 0,001 \quad 2 \mathrm{~h}$ postquirúrgicas

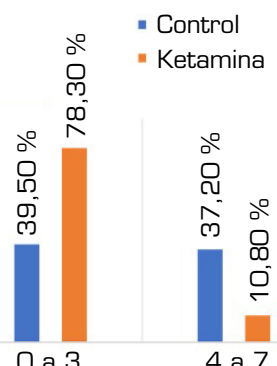

$p 0,391$

2-24 h postquirúrgicas

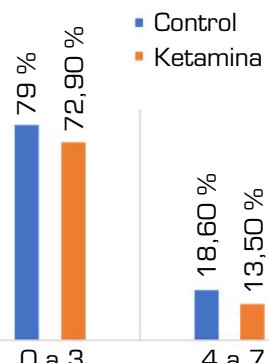

$p 0,612$

$48 \mathrm{~h}$ postquirúrgicas

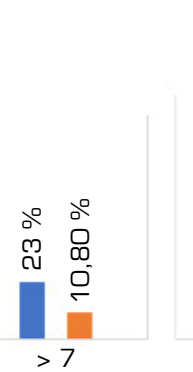

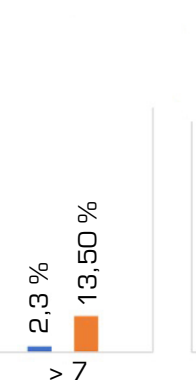

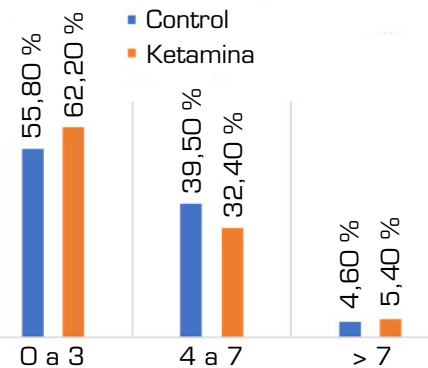

Fig. 2. Niveles de EVN durante las $48 \mathrm{~h}$ postoperatorias. Prueba $\mathrm{U}$ de Mann-Whitney.

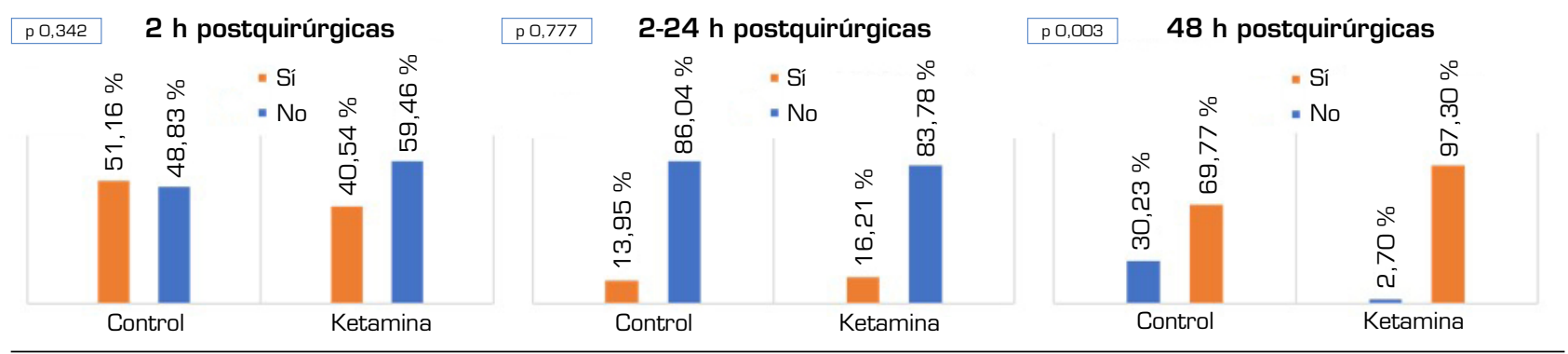

Fig. 3. Porcentaje de pacientes que necesitaron o no la administración de bolus puntales de morfina. Prueba Chi cuadrado.

la perfusión continua intraoperatoria de ketamina puede evitar el componente de hiperalgesia actuando sobre los receptores NMDA [20]. En nuestro centro, las necesidades intraoperatorias de opioides pueden llegar a alcanzar los $1100 \mathrm{mcg}$ de fentanilo intravenoso. No se ha analizado si el factor principal que determina la necesidad de dosis tan elevadas es la mayor agresión quirúrgica o si el tratamiento con opioides de forma crónica es un factor relevante. En los pacientes del grupo ketamina, a pesar de presentar necesidades de opioides intraoperatorias similares a los del grupo control, los registros de EVN durante las dos primeras horas postoperatorias son menores de forma significativa. El efecto de la perfusión a dosis subanestésicas de ketamina durante la cirugía podría justificar este hallazgo. 


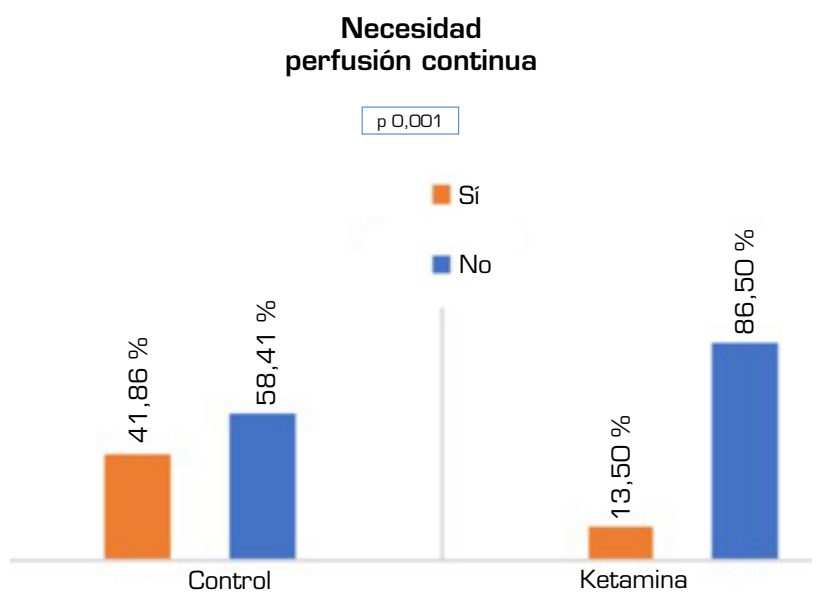

Fig. 4. Necesidad de perfusión de morfina durante desde el ingreso en reanimación hasta las 48 h postquirúrgicas. Prueba Chi cuadrado.

En nuestro centro, en las primeras $24 \mathrm{~h}$ postquirúrgicas los pacientes no se movilizan. El control del dolor en este periodo es aceptable en ambos grupos. Sin embargo, a las 48 horas comienza la sedestación y movilización precoz. En el caso del grupo ketamina, los pacientes presentan un mejor control analgésico basal, necesitando menos opioides de rescate. El mantenimiento de la ketamina en este periodo puede ser clave, ya que el estímulo nociceptivo es, de nuevo, de gran intensidad (12).

Este análisis presenta algunas limitaciones. Durante el periodo postoperatorio, no fue posible recoger el número de bolus de PCA de morfina o PCA de morfina + ketamina administrados por cada paciente. Tampoco las dosis de morfina de rescate administrada en la planta de hospitalización. Asimismo, desconocemos si los registros de EVN son en movimiento o en reposo.

\section{CONCLUSIÓN}

El uso de la ketamina durante el intraoperatorio y las primeras $48 \mathrm{~h}$ postoperatorias en pacientes con tratamiento crónico con opioides sometidos a cirugía espinal disminuye las necesidades de opioides postoperatorios y mejora el control del dolor las primeras 2 horas postoperatorias. En un futuro, la realización de un estudio observacional prospectivo nos posibilitaría conocer de forma exhaustiva el control del dolor, la necesidad de opioides de rescate, el grado de satisfacción y la tasa de complicaciones en los pacientes en tratamiento con ketamina. Sería interesante observar el efecto de dicho fármaco tras 6 y 12 meses de la intervención, ya que, el uso de ketamina intraoperatoria podría disminuir el dolor a largo plazo y la cronificación del mismo.

\section{CONFLICTO DE INTERESES}

Los autores declaran no tener ningún conflicto de intereses.
ANEXO 1

TABLA DE EQUIVALENCIA DE OPIOIDES

\begin{tabular}{|l|l|}
\hline \multicolumn{1}{|c|}{ Fármaco } & \multicolumn{1}{|c|}{$\begin{array}{c}\text { Dosis equivalente a 30 } \\
\text { mg de morfina oral }\end{array}$} \\
\hline Morfina & $30 \mathrm{mg}$ \\
\hline Codeína & $300 \mathrm{mg}$ \\
\hline Tramadol & $300 \mathrm{mg}$ \\
\hline Fentanilo & $\begin{array}{l}12,5 \mathrm{mcg} / \mathrm{h} \\
\text { (transdérmico) }\end{array}$ \\
\hline Hidromorfona & $4 \mathrm{mg}$ \\
\hline Buprenorfina & $\begin{array}{l}12,5 \mathrm{mcg} / \mathrm{h} \\
\text { (transdérmico) }\end{array}$ \\
\hline Oxicodona & $15 \mathrm{mg}$ \\
\hline Metadona & Variable * \\
\hline Tapentadol & $100 \mathrm{mg}$ \\
\hline
\end{tabular}

Fuente: O`Brien T, Christrup LL, Drewes AM, Fallon MT, Kress HG. European Pain Federation position paper on appropriate opioid use in chronic pain management. Eur J Pain. 2017;21(1):3-19. DOI: 10.1002/ejp.970.

* Uso de 5:1 para conversión de metadona a morfina oral. (Fuente: Mercadante S, Casucio A, Calderone $\mathrm{L}$. Rapid switching from morphine to methadone in cancer patients with poor response to morphine. J Clin Oncol. 1999;17[10]:3307-12. DOI: 10.1200/ JCO.1999.17.10.3307.).

\section{BIBLIOGRAFÍA}

1. Polanco-García M, García-Lopez J, Fábregas N, Meissner W, Puig MM, Consortium POS. Postoperative Pain Management in Spanish Hospitals: A Cohort Study Using the PAIN-OUT Registry. J Pain. 2017;18(10):1237-52. DOl: 10.1016/j. jpain.2017.05.006.

2. Kim HJ, Park JH, Kim JW, Kang KT, Chang BS, Lee CK, et al. Prediction of postoperative pain intensity after lumbar spinal surgery using pain sensitivity and preoperative back pain severity. Pain Med. 2014;15(12):2037-45. DOI: 10.1111/ pme.12578.

3. Jain N, Phillips FM, Weaver T, Khan SN. Preoperative Chronic Opioid Therapy: A Risk Factor for Complications, Readmission, Continued Opioid Use and Increased Costs After One- and Two-Level Posterior Lumbar Fusion. Spine (Phila Pa 1976). 2018;43(19):1331-8. DOI: 10.1097/ BRS.0000000000002609.

4. Walid MS, Hyer L, Ajjan M, Barth AC, Robinson JS. Prevalence of opioid dependence in spine surgery patients and correlation with length of stay. J Opioid Manag. 2007;3(3):127-8, 30-2.

5. Patanwala AE, Jarzyna DL, Miller MD, Erstad BL. Comparison of opioid requirements and analgesic response in opioid-tolerant versus opioid-naÔve patients after total knee arthroplasty. Pharmacotherapy. 2008;28(12):1453-60. DOI: 10.1592/phco.28.12.1453.

6. Chou R, Gordon DB, de Leon-Casasola OA, Rosenberg JM, Bickler S, Brennan T, et al. Management of Postoperative Pain: A Clinical Practice Guideline From the American Pain Society, the American Society of Regional Anesthesia and Pain Medicine, and the American Society of Anesthesiologists' Committee on Regional Anesthesia, Executive Commit- 
tee, and Administrative Council. J Pain. 2016;17(2):13157. DOl: 10.1016/j.jpain.2015.12.008.

7. Schwenk ES, Viscusi ER, Buvanendran A, Hurley RW, Wasan $A D$, Narouze $S$, et al. Consensus Guidelines on the Use of Intravenous Ketamine Infusions for Acute Pain Management From the American Society of Regional Anesthesia and Pain Medicine, the American Academy of Pain Medicine, and the American Society of Anesthesiologists. Reg Anesth Pain Med. 2018;43(5):456-66. DOI: 10.1097/ AAP.0000000000000806.

8. Maher C, Underwood M, Buchbinder R. Non-specific low back pain. Lancet. 2017;389(10070):736-47. DOI: 10.1016/ S0140-6736(16)30970-9.

9. Carames M, Robaina F, Calvo B. Opioides en el dolor raquídeo. Relación riesgo/beneficio y estrategia apropiada para su utilización. Rev Soc Esp Dolor. 2010;17(3):169-76. DOI: 10.1016/S1134-8046(10)70027-6.

10. Deyo RA, Von Korff M, Duhrkoop D. Opioids for low back pain. BMJ. 2015;350:g6380. DOl: 10.1136/bmj.g6380.

11. Mugabure Bujedo B, Gonzalez Santos S, Tranque Bizueta I, Araujo Lopez A, Toran Garcia L. Manejo del dolor perioperatorio de los pacientes en tratamiento crónico con opioides. Rev Soc Esp Dolor. 2009;16(5):288-97. DOI: 10.1016/ S1134-8046(09)72038-5.

12. Himmelseher S, Durieux ME. Ketamine for perioperative pain management. Anesthesiology. 2005;102(1):211-20. DOI: 10.1097/00000542-200501000-00030.

13. Garg N, Panda NB, Gandhi KA, Bhagat H, Batra YK, Grover VK, et al. Comparison of Small Dose Ketamine and Dexmedetomidine Infusion for Postoperative Analgesia in Spine Surgery-A Prospective Randomized Double-blind Placebo Controlled Study. J Neurosurg Anesthesiol. 2016;28(1):2731. DOI: 10.1097/ANA.0000000000000193.
14. Loftus RW, Yeager MP, Clark JA, Brown JR, Abdu WA, Sengupta DK, et al. Intraoperative ketamine reduces perioperative opiate consumption in opiate-dependent patients with chronic back pain undergoing back surgery. Anesthesiology. 2010;113(3):639-46. DOI: 10.1097/ ALN.Ob013e3181e90914.

15. Nielsen RV, Fomsgaard JS, Siegel H, Martusevicius R, Nikolajsen L, Dahl JB, et al. Intraoperative ketamine reduces immediate postoperative opioid consumption after spinal fusion surgery in chronic pain patients with opioid dependency: a randomized, blinded trial. Pain. 2017;158(3):463-70. DOI: 10.1097/j.pain.0000000000000782.

16. Carroll IR, Angst MS, Clark JD. Management of perioperative pain in patients chronically consuming opioids. Reg Anesth Pain Med. 2004;29(6):576-91. DOI: 10.1016/j. rapm.2004.06.009.

17. Kim D, Lim HS, Kim MJ, Jeong W, Ko S. High-dose intraoperative remifentanil infusion increases early postoperative analgesic consumption: a prospective, randomized, doubleblind controlled study. J Anesth. 2018;32(6):886-9. DOI: 10.1007/s00540-018-2569-6.

18. Chia YY, Liu K, Wang JJ, Kuo MC, Ho ST. Intraoperative high dose fentanyl induces postoperative fentanyl tolerance. Can J Anaesth. 1999;46(9):872-7. DOI: 10.1007/ BFO3012978.

19. Hansen EG, Duedahl TH, $\mathrm{R}^{-}$msing J, Hilsted KL, Dahl JB. Intra-operative remifentanil might influence pain levels in the immediate post-operative period after major abdominal surgery. Acta Anaesthesiol Scand. 2005;49(10):1464-70. DOl: 10.1111/j.1399-6576.2005.00861.x.

20. Peltoniemi MA, Hagelberg NM, Olkkola KT, Saari TI. Ketamine: A Review of Clinical Pharmacokinetics and Pharmacodynamics in Anesthesia and Pain Therapy. Clin Pharmacokinet. 2016;55(9):1059-77. DOI: 10.1007/s40262-016-0383-6. 